\title{
Controle do tabagismo em instituição de longa permanência para idosos: relato de experiência
}

\author{
Campaign against smoking in a home for the elderly: \\ the report of an experiment
}

Anderson Albuquerque de Carvalho ${ }^{1}$

Lucy Gomes ${ }^{1}$

Altair Lahud Loureiro ${ }^{1}$

Armando José China Bezerra ${ }^{1}$

${ }^{1}$ Programa de Pósgraduação Stricto Sensu em Gerontologia, Universidade Católica de Brasília. SGAN 916 Módulo B W5, Asa Norte. 70790-160 Brasília DF.

carvalhofisio2003@gmail.com

\begin{abstract}
Studies on institutionalized elderly smokers are important for developing appropriate preventive measures. This was a cross-sectional population-based study of individuals over 60 admitted to a Home for the Elderly in the Federal District. The following aspects were investigated: smoking prevalence, socio-demographic and clinical profile, gender, education, probable depression, degree of motivation to stop smoking, prior use of medication to stop smoking and perception of the harm of smoking to health. Spirometry, the measurement of exhaled carbon monoxide and classification according to severity of nicotine dependence were conducted. The program followed guidelines for stopping smoking, and was evaluated one year later. Results revealed that $25.7 \%$ were smokers, $22.8 \%$ men and $2.9 \%$ women, mean age $68.3 \pm 8.5$ years. A significant decrease in smoking among the more elderly was detected. Significant associations emerged between the degree of nicotine dependence and education, probable depression, degree of motivation for stopping smoking and perception of the harm of smoking to health. Higher indices of carbon monoxide were identified in individuals with severe obstruction. Initially, 37.9\% of smokers agreed to participate in the campaign, and after a year 9\% of them succeeded in stopping smoking.

Key words Home for the elderly, Campaign against smoking, Elderly, Smokers
\end{abstract}

Resumo Estudos sobre tabagismo em idosos institucionalizados são importantes, para que se desenvolvam intervenções apropriadas. Estudo transversal, base populacional, em indivíduos com 60 anos ou mais, internados em ILPI do DF. Verificou-se prevalência de tabagismo, perfil sociodemográfico e clínico: gênero, escolaridade, provável depressão, grau de motivação para cessação tabágica, uso anterior de auxílio medicamentoso para abolição do tabagismo e percepção de prejuízo do fumo para a saúde. Realizadas espirometria, medição do monóxido de carbono exalado e classificação segundo gravidade da dependência nicotínica. O Programa seguiu diretrizes para cessação do tabagismo, sendo avaliado após um ano do início. Do total, $25,7 \%$ eram fumantes, sendo $22,8 \%$ homens e 2,9\% mulheres, com idade média 68,3 \pm 8,5 anos. Surgiu significativa redução da prevalência de tabagismo nos mais idosos e associações significativas entre grau de dependência nicotínica e escolaridade, provável depressão, grau de motivação para cessação de tabagismo e percepção de prejuízo do fumo para saúde. Identificaram-se maiores valores de monóxido de carbono naqueles com obstrução grave. Inicialmente, 37,9\% dos tabagistas concordaram em participar da Campanha, obtendo-se êxito, após um ano, em 9\% deles. Palavras-chave Instituição de Longa Permanência para Idosos, Campanhas para Controle do Tabagismo, Idosos, Tabagismo 


\section{Introdução}

O uso de tabaco entre indivíduos idosos é problema de saúde importante e potencialmente previsível. No Brasil, há cerca de um milhão a um milhão e meio de idosos fumantes ${ }^{1}$. Na região sul do país esta prevalência foi de $10,6 \%$ e no município de Bambuí, Minas Gerais, foi de $18,7 \%^{2,3}$

Entre os idosos, idêntico ao encontrado no grupo de adultos, o tabagismo predomina nos homens ${ }^{4}$. A prevalência de tabagismo nos homens e mulheres idosos foi de $19,6 \%$ e $8,1 \%$ na região metropolitana de Belo Horizonte e de $31,4 \%$ e $8,1 \%$ no município de Bambuí, respectivamente ${ }^{5}$. Estudo realizado em 15 capitais brasileiras encontrou prevalência de tabagismo nos idosos entre 8,6\% (em Vitória) e 24,1\% (em São Paulo) nos homens e 2,8\% (em Belo Horizonte) e 11,1\% (em Campo Grande) nas mulheres. No Distrito Federal, esta prevalência foi de 15,9\% nos homens idosos e 9,9\% nas mulheres idosas ${ }^{6}$.

O tabagismo em indivíduos idosos tem recebido pouca atenção ${ }^{7}$, devido ao pressuposto de que a pessoa por estar em idade avançada merece o último prazer, que seria o de fumar ${ }^{8}$. Assim, o idoso fumante tem sido excluído dos Programas de Cessação Tabágica, em detrimento ao quase exclusivo direcionamento dos mesmos às populações adolescente e adulta. Entretanto, a idade avançada não é fator limitante na aplicação dos procedimentos para a supressão do tabaco ${ }^{9}$. Diversos benefícios podem ser obtidos com a abolição do tabagismo em indivíduos idosos e mesmo naqueles muito idosos, destacando-se: redução do risco de adoecer, melhor controle da evolução de doença pré-existente, melhor qualidade de vida e aumento na expectativa de vida ${ }^{10-12}$. Nos idosos que fumam até um maço de cigarros por dia, há aumento de dois a três anos na expectativa de vida após abandono do tabagismo ${ }^{13}$.

Os fatores que afetam o uso do tabaco entre os idosos não são completamente compreendidos. Entre as barreiras para o sucesso da cessação tabágica neste grupo etário, estão as altas taxas de dependência nicotínica apresentadas ${ }^{14} \mathrm{e}$, frequentemente, a submissão a situações de estresse e de isolamento social ${ }^{15}$.

A dependência nicotínica é constituída de complexa relação entre estímulos ambientais, hábitos pessoais, condicionamentos psicossociais e ações biológicas da nicotina. Nesse contexto, a sua inserção nos indivíduos internados em Instituições de Longa Permanência para Idosos (ILPI) compreende campo de estudo diferenciado, vis- to que os processos patológicos que acometem os idosos precipitam-se no interior das ILPI, pois a institucionalização frequentemente contribui para maior isolamento e inatividade física e mental, provocando consequências negativas à saú$\mathrm{de}^{16-18}$. Portanto, é pertinente identificar essas peculiaridades, a fim de inserir essa população no processo contínuo e dinâmico de promoção da saúde e melhoria na qualidade de vida ${ }^{19}$.

É necessário que se obtenham informações sobre o consumo de tabaco em grupos vulneráveis como o de idosos institucionalizados, para que se desenvolvam nos mesmos as intervenções apropriadas. Identifica-se lacuna a ser preenchida na literatura científica referente às possíveis particularidades da população idosa tabagista internada em ILPI.

Visto que a causa do tabagismo é multifacetada e multifatorial, o idoso institucionalizado pode responder às diferentes influências do meio no qual está inserido de maneira não análoga ao da população de idosos não institucionalizados. Entender os valores, as crenças, as atitudes e os comportamentos relacionados ao tabagismo em idosos institucionalizados pode ajudar a desenvolver estratégias que reduzam a morbidade e a mortalidade associadas.

O objetivo do presente estudo foi avaliar a prevalência de tabagismo em idosos internados em ILPI do Distrito Federal (DF), levantar dados acerca do perfil sociodemográfico e clínico dos mesmos e implementar Campanha de Cessação Tabágica entre esses indivíduos.

\section{Métodos}

Trata-se de estudo transversal com base populacional, realizado de janeiro a dezembro de 2008, em indivíduos com idade igual ou superior a 60 anos, internados na ILPI "Parque Assistencial Jorge Cauhy", situada no Núcleo Bandeirante, DF. Esta ILPI é credenciada no Conselho dos Direitos do Idoso, constituindo-se como instituição filantrópica conveniada e subsidiada pela Secretaria de Estado de Ação Social do DF. Neste local são internados idosos sem renda econômica ou recebendo um salário mínimo, que não possuem família ou esta não tem condições financeiras de mantê-los. Aqueles com distúrbios psiquiátricos acompanhados de agressividade não são admitidos.

A ILPI estudada possui área total de 40.000 $\mathrm{m}^{2}$, com $12.000 \mathrm{~m}^{2}$ de área construída. Não há locais destinados exclusivamente aos usuários de 
tabaco, sendo este utilizado nas diferentes dependências da instituição, entre elas: áreas de uso comum, como varandas, corredores e praças; e áreas de uso mais restrito, como dormitórios e banheiros dos quartos. Identificou-se, nesse contexto, que os idosos não tabagistas encontravamse expostos à fumaça dos cigarros utilizados pelos indivíduos tabagistas, tornando-se tabagistas passivos ${ }^{1}$.

Inicialmente, em todos os idosos tabagistas foi aplicado o Mini Exame do Estado Mental ${ }^{20}$, utilizando-se ponto de corte segundo a escolaridade ${ }^{21}$.

Identificou-se a prevalência de tabagismo nos idosos institucionalizados. Nos idosos tabagistas foram pesquisados os perfis sociodemográfico e clínico: gênero, escolaridade, provável depressão, grau de motivação para cessação tabágica, uso anterior de auxílio medicamentoso para abolição do tabagismo e percepção de prejuízo do fumo para a saúde.

A avaliação de provável depressão foi feita utilizando-se a Escala de Depressão Geriátrica ${ }^{22}$, na versão reduzida com 15 itens $^{23}$.

Os idosos tabagistas foram classificados em três grupos, segundo a gravidade da dependência nicotínica, medida através do Teste de Fagerström ${ }^{24}$, validado no Brasil por Meneses-Gaya et al. ${ }^{25}$. Relacionou-se a gravidade da dependência nicotínica com o perfil sociodemográfico e o clínico desses idosos.

Realizada espirometria completa nos idosos tabagistas, empregando-se espirômetro portátil, Microlab 3500, Micro Medical Ltda, Reino Unido. Utilizaram-se como valores normais os preconizados por Pereira et al. ${ }^{26}$. A medida da função pulmonar possibilitou diagnosticar os pacientes portadores de doença pulmonar obstrutiva crônica (DPOC), assim como estadiar a gravidade desta patologia ${ }^{27}$.

Feita a medição do monóxido de carbono exalado (COex) nos idosos tabagistas, com aparelho MicroCO Meter, Micro Medical Ltd., Rochester, Reino Unido, a fim de verificar o seu grau de exposição à fumaça do cigarro. Esse aparelho mede a concentração de COex através de um sensor eletroquímico, expressando-a em partes por milhão (CO-PPM) e em porcentual $(\mathrm{CO} \%)^{28}$. O exame foi realizado antes do início da Campanha de Cessação Tabágica e repetido um ano após seu início naqueles que se mantiveram sem fumar.

O Programa de Controle do Tabagismo executado na ILPI caracterizou-se por desenvolver ações sincronizadas e contínuas, com intervenções nos âmbitos educativo, físico e terapêutico, seguindo as diretrizes para cessação do tabagis$\mathrm{mo}^{29}$. Foram efetuadas as seguintes intervenções: 1. educativas: palestras sobre tabagismo (informando-se sobre seus riscos e benefícios da supressão, com possibilidade de sucesso sem sofrimento intenso), cartazes sobre tabagismo afixados nos murais e folhetos versando sobre seu controle; 2. físicas: delimitação de áreas para fumar (fumódromos), sinalização com adesivos e placas com aviso de proibido fumar, remoção de cinzeiros dos ambientes fechados, remoção de materiais de cunho promocional do tabaco e remanejamento dos idosos tabagistas para dormitórios distintos dos não tabagistas; 3 . terapêuticas: terapia farmacológica nicotínica (adesivo de nicotina-TRN) e não nicotínica (bupropiona) e abordagem cognitivo-comportamental, realizada em quatro sessões semanais de $90 \mathrm{mi}-$ nutos, seguidas de duas sessões quinzenais de 60 minutos e uma sessão mensal de acompanhamento até completar um ano, com o fim de prevenir recaídas. $\mathrm{O}$ uso e a posologia das terapias farmacológicas seguiram a classificação da dependência nicotínica ${ }^{30}$.

Avaliou-se a Campanha de Cessação Tabágica após um ano do início da mesma, contabilizandose os idosos que permaneceram sem fumar ${ }^{31}$.

Os dados obtidos foram analisados por meio de estatística descritiva, com média e desvio padrão. A variável dependente utilizada foi o grau de dependência nicotínica. A associação entre a variável dependente e as variáveis independentes estudadas foi verificada na análise bivariada pelo teste qui-quadrado $\left(\chi^{2}\right)$, com nível de significância de $5 \%$. Na análise multivariada, foram consideradas as variáveis que tiveram valor de $\mathrm{p}$ inferior a 0,20 na análise bivariada, permanecendo no modelo apenas aquelas com $\mathrm{p}<0,05$. Foram associados os resultados da espirometria e da concentração de COex, utilizando-se o teste de Pearson. A análise estatística foi feita com o software Statistical Package for the Social Sciences, versão 14.0 (SPSS Inc, Chicago, IL, EUA) para Windows ${ }^{\circledR}$.

\section{Resultados}

Encontraram-se 136 idosos internados na ILPI, sendo $69(50,7 \%)$ homens e 67 (49,3\%) mulheres, com idade média de 69,9 \pm 7,2 anos. Destes, $35(25,7 \%)$ eram fumantes, sendo $31(22,8 \%)$ homens e $4(2,9 \%)$ mulheres $(p=0,042)$. A média de idade da população tabagista foi de $68,3 \pm$ 8,5 anos. Todos os idosos tabagistas apresenta- 
ram o Mini Exame do Estado Mental dentro dos limites da normalidade.

As frequências das variáveis sociodemográficas e clínicas encontradas na população tabagista estão descritas na Tabela 1.

Surgiu significativa diminuição da prevalência de tabagismo nas faixas etárias mais avançadas $(\mathrm{p}=0,041)$.

Não surgiram associações significativas entre o grau de dependência nicotínica e as seguintes variáveis: gênero, faixa etária e uso anterior de auxílio medicamentoso para cessação tabágica.

A dependência nicotínica leve ocorreu mais frequentemente nas faixas etárias mais elevadas

Tabela 1. Distribuição das variáveis sóciodemográficas e de saúde nos 35 idosos tabagistas institucionalizados no "Parque Assistencial Jorge Cauhy", 2008.

\begin{tabular}{|c|c|c|}
\hline Variáveis & $\mathrm{N}^{0}$ & $\%$ \\
\hline \multicolumn{3}{|l|}{ Gênero } \\
\hline Masculino & 31 & 88 \\
\hline Feminino & 4 & 11 , \\
\hline \multicolumn{3}{|l|}{ Faixa etária } \\
\hline 60 a 69 anos & 17 & 48,6 \\
\hline 70 a 79 anos & 15 & 42,9 \\
\hline 80 anos ou mais & 3 & \\
\hline \multicolumn{3}{|l|}{ Escolaridade } \\
\hline Nunca foi a escola & 12 & 34,2 \\
\hline 1 a 3 anos & 13 & 37,2 \\
\hline 4 a 7 anos & 6 & 17,2 \\
\hline 8 a 11 anos & 2 & 5,7 \\
\hline 12 anos ou mais & 2 & 5,7 \\
\hline \multicolumn{3}{|l|}{ Provável depressão } \\
\hline Sim & 23 & 65,7 \\
\hline Não & 12 & 34,3 \\
\hline \multicolumn{3}{|c|}{$\begin{array}{l}\text { Grau de motivação para cessação } \\
\text { do tabagismo }\end{array}$} \\
\hline Baixa & 15 & 42,9 \\
\hline Moderada & 9 & 25,7 \\
\hline Alta & 11 & 31,4 \\
\hline \multicolumn{3}{|c|}{ Grau de dependência nicotínica } \\
\hline Leve & 14 & 40,0 \\
\hline Moderada & 10 & 28,6 \\
\hline Elevada & 11 & 31,4 \\
\hline \multicolumn{3}{|c|}{$\begin{array}{l}\text { Uso anterior de auxílio } \\
\text { medicamentoso para cessação } \\
\text { tabágica }\end{array}$} \\
\hline Sim & 2 & 5,7 \\
\hline Não & 33 & 94,3 \\
\hline \multicolumn{3}{|c|}{$\begin{array}{l}\text { Percepção do prejuízo do fumo para } \\
\text { saúde }\end{array}$} \\
\hline $\operatorname{Sim}$ & 16 & 45,7 \\
\hline Não & 19 & 54,3 \\
\hline
\end{tabular}

(70 a 79 e 80 anos e mais), tendo surgido a mesma proporção de idosos com dependência leve e moderada na faixa de 60 a 69 anos, não havendo associação significativa entre a dependência e esta variável $(\mathrm{p}=0,2)$.

Verificaram-se associações significativas entre o grau de dependência nicotínica e as seguintes variáveis: escolaridade $(\mathrm{p}=0,01)$, provável depressão $(\mathrm{p}=0,01)$, motivação para cessação de tabagismo $(p=0,007)$ e percepção de prejuízo do fumo para a saúde $(\mathrm{p}=0,02)$ (Tabela 2). No modelo multivariado, as variáveis que permaneceram significativas foram: provável depressão $(\mathrm{p}=0,01)$, grau de motivação para a cessação de tabagismo $(\mathrm{p}=0,001)$ e percepção de prejuízo do fumo para a saúde $(p=0,02)$. Identificou-se, assim, aumento significativo de dependência nicotínica à medida que diminuiu a escolaridade $\mathrm{e}$ aumentaram as frequências de provável depressão, de motivação para cessação de tabagismo e de percepção de prejuízo do fumo para a saúde. Surgiu provável depressão em $63 \%$ dos idosos tabagistas institucionalizados, predominando significativamente naqueles com maior grau de dependência nicotínica $(p=0,01)$.

Foram avaliadas função pulmonar e concentração de COex em 29 dos 35 idosos tabagistas. Estas perdas foram consequentes a: duas por óbitos, duas por internações hospitalares e duas por transferências para outras ILPI. A função pulmonar e a concentração de COex nestes idosos estão descritas na Tabela 3.

$\mathrm{Na}$ espirometria, os 29 idosos tabagistas apresentaram predominância de padrão obstrutivo (79,3\% dos casos), enquanto o padrão restritivo foi observado em 10,3\% deles. Os restantes $10,3 \%$ exibiram a espirometria normal. Entre aqueles com distúrbio obstrutivo, $31,0 \%$ os mostraram em grau leve, $20,7 \%$ em grau moderado e $27,6 \%$ em grau severo.

Identificaram-se maiores valores de CO-PPM e CO\% nos idosos com distúrbio obstrutivo grave, não se observando esta associação naqueles com distúrbio restritivo $(\mathrm{p}=0,02)$. Os seis idosos que apresentaram distúrbio obstrutivo em grau leve tiveram COex médio de 0,45 , os oito com este distúrbio em grau moderado obtiveram 0,96 e os nove em grau severo alcançaram 2,08 . Os três idosos que mostraram a espirometria normal tiveram o valor médio de 0,36 e os três com distúrbio restritivo de 0,59 (Tabela 3 ).

Entre os 29 idosos tabagistas, inicialmente 11 $(37,9 \%)$ concordaram em participar da Campanha de Cessação Tabágica. Na Tabela 4 estão descritos o primeiro mês de tratamento, com as 
quatro seções semanais iniciais, e a primeira sessão de manutenção quinzenal.

$\mathrm{Na}$ segunda semana da Campanha houve desistência de um dos integrantes do grupo, na terceira semana de três componentes e, na quarta, de mais um deles. Das cinco perdas, quatro foram consequentes à intolerância aos sintomas de abstinência e uma por hospitalização. Na primeira seção de manutenção quinzenal, entre os 11 idosos que iniciaram a Campanha apenas cinco continuaram a participar, estando todos eles em uso de terapia medicamentosa (bupropiona isolada em três e associada a TRN em dois). Destes, dois mantiveram-se sem fumar no período, enquanto os outros três somente reduziram o número de cigarros-dia.

$\mathrm{Na}$ segunda reunião quinzenal de manutenção, identificaram-se mais três desistências, mantendo-se apenas dois integrantes do grupo inicial. Este fato perdurou até a quarta seção de ma- nutenção, perfazendo três meses do início da Campanha. A partir desse momento, os dois integrantes que mantiveram a abstinência ao tabaco tiveram suspenso o auxílio medicamentoso. No $12^{\circ}$ mês após o início da Campanha, constatou-se que apenas um dos idosos manteve-se sem fumar, fato confirmado com a ausência de COPPM e CO\% no ar expirado do mesmo. Assim, a Campanha de Cessação Tabágica obteve êxito em $9 \%$ da população de idosos tabagistas institucionalizados.

Durante o uso da terapia medicamentosa com bupropiona não foram identificados efeitos adversos. Todavia, com o uso da TRN foram relatados prurido de curta duração (inferior a duas hs), no local de fixação do adesivo em cinco $(83,3 \%)$ e insônia em três (50\%), não havendo necessidade de suspensão da medicação em nenhum desses idosos.

Tabela 2. Associação entre o grau de dependência nicotínica e variáveis sociodemográficas e de saúde nos 35 idosos tabagistas institucionalizados no "Parque Assistencial Jorge Cauhy", 2008.

\begin{tabular}{|c|c|c|c|c|c|}
\hline \multirow[b]{2}{*}{ Variáveis } & \multicolumn{3}{|c|}{ Dependência Nicotínica } & \multirow[b]{2}{*}{$\begin{array}{c}\text { Total } \\
\mathbf{N}^{\circ}\end{array}$} & \multirow[b]{2}{*}{ Valor $\mathrm{p}$} \\
\hline & $\begin{array}{c}\text { Baixa } \\
\mathrm{N}^{\circ}(\%)\end{array}$ & $\begin{array}{l}\text { Moderada } \\
\mathrm{N}^{\circ}(\%)\end{array}$ & $\begin{array}{l}\text { Elevada } \\
\mathrm{N}^{\circ}(\%)\end{array}$ & & \\
\hline Gênero & & & & & 0,9 \\
\hline Masculino & $12(38,7)$ & $9(29,0)$ & $10(32,3)$ & 31 & \\
\hline Feminino & $2(50,0)$ & $1(25,0)$ & $1(25,0)$ & 4 & \\
\hline Faixa Etária & & & & & 0,2 \\
\hline 60 a 69 anos & $6(35,3)$ & $6(35,3)$ & $5(29,4)$ & 17 & \\
\hline 70 a 79 anos & $6(40,0)$ & $4(26,7)$ & $5(33,3)$ & 15 & \\
\hline 80 anos ou mais & $2(66,7)$ & & $1(33,3)$ & 3 & \\
\hline Escolaridade & & & & & $0,01^{*}$ \\
\hline Nunca foi à escola & $4(33,3)$ & $4(33,3)$ & $4(33,3)$ & 12 & \\
\hline 1 a 3 anos & $1(7,8)$ & $6(46,1)$ & $6(46,1)$ & 13 & \\
\hline 4 a 7 anos & $6(100,0)$ & - & - & 6 & \\
\hline 8 a 11 anos & $2(100,0)$ & - & - & 2 & \\
\hline 12 anos ou mais & $1(50,0)$ & - & $1(50,0)$ & 2 & \\
\hline Provável depressão & & & & & $0,01^{*}$ \\
\hline Sim & $5(22,7)$ & $7(31,8)$ & $10(45,5)$ & 22 & \\
\hline Não & $9(69,2)$ & $3(23,1)$ & $1(7,7)$ & 13 & \\
\hline Grau de motivação para cessação tabágica & & & & & $0,007^{*}$ \\
\hline Leve & $2(13,3)$ & $5(33,3)$ & $8(53,3)$ & 15 & \\
\hline Moderada & $3(33,3)$ & $4(44,4)$ & $2(22,2)$ & 9 & \\
\hline Elevada & $9(81,8)$ & $1(9,1)$ & $1(9,1)$ & 11 & \\
\hline $\begin{array}{l}\text { Uso anterior de auxílio medicamentoso para } \\
\text { cessação do tabagismo }\end{array}$ & & & & & 0,4 \\
\hline Sim & - & $1(50,0)$ & $1(50,0)$ & 2 & \\
\hline Não & $14(42,4)$ & $9(27,3)$ & $10(30,3)$ & 33 & \\
\hline Percepção de prejuízo do fumo para saúde & & & & & $0,02^{*}$ \\
\hline Sim & $11(61,1)$ & $4(22,2)$ & $3(16,7)$ & 18 & \\
\hline Não & $3(17,6)$ & $6(35,3)$ & $8(47,1)$ & 17 & \\
\hline
\end{tabular}

${ }^{*}$ significativo 


\section{Discussão}

A prevalência de tabagismo entre os idosos internados na ILPI estudada foi de $25,7 \%$. A maioria dos estudos que faz menção à prevalência de tabagismo em população idosa refere-se a indivíduos vivendo na comunidade. Nessas pesquisas em idosos não institucionalizados, as prevalências relatadas foram iguais ou maiores que $14 \%$, 32 .

Foram identificados dois estudos abordando a prevalência de tabagismo em idosos institucionalizados. Nos mesmos, Davim et al. ${ }^{18}$ mostraram a prevalência de $14 \%$, enquanto Gonçalves et al. ${ }^{33}$ relataram-na sendo de $11 \%$. Provavelmente, as menores prevalências expostas nestas pesquisas, em relação à encontrada no estudo atual, devem-se às diferenças na distribuição de gêneros nas populações estudadas. Surgiram menores prevalências de tabagismo nos estudos

cujas amostras foram formadas predominantemente por mulheres idosas ( $64 \%$ e $75 \%$ nos dois estudos citados, respectivamente). No presente estudo, somente $2,9 \%$ dos idosos tabagistas institucionalizados eram do gênero feminino. A menor prevalência tabágica nas mulheres vem de encontro ao perfil sociocultural desses indivíduos, no qual o homem fuma mais enquanto jovem e permanece fumando mais quando comparado à mulher idosa de faixa etária análoga ${ }^{18}$. Entretanto, em estudo realizado em 13 ILPI do DF, encontramos a prevalência tabágica de $23 \%$, sendo $24 \%$ nos homens idosos e $20,1 \%$ nas mulheres idosas ${ }^{34}$. Há a necessidade de futuros estudos para averiguar as causas deste achado, pois encontrou-se maior prevalência de tabagismo nos idosos internados nas ILPI do DF do que as descritas em ILPI de outras localizações do país.

No estudo atual, a prevalência de tabagismo foi de $48,6 \%$ na faixa etária de 60 a 69 anos, sendo de apenas $8,6 \%$ naqueles com 80 anos ou mais, com diferença significativa. Tal fato está de acordo com os achados de outros autores ${ }^{5,32}$ que também demonstraram diminuição da prevalência tabágica com o aumento da faixa etária. Entretanto, mais uma vez devemos realçar que as pesquisas anteriores abordaram idosos não institucionalizados.

No presente estudo, os idosos tabagistas institucionalizados recebiam, no máximo, um salário mínimo. No Brasil, o consumo de cigarros é maior nas classes de menor rendimento ${ }^{35}$, corroborando o achado de alta prevalência tabágica nos idosos estudados.

Nos idosos tabagistas de ambos os gêneros encontrou-se maior frequência de dependência nicotínica leve, sendo a dependência elevada preponderante no gênero masculino, embora sem diferença significativa. Todavia, a disparidade na proporção de homens e mulheres tabagistas institucionalizados $(22,8 \%$ homens e $2,9 \%$ mulheres) pode justificar este achado.

Não foi significativa a associação entre grau de dependência nicotínica e faixa etária, tendo-se encontrado maior frequência de dependência leve nas faixas etárias mais elevadas. Este achado é discordante dos relatados anteriormente, nos quais o maior tempo de exposição do organismo à nicotina levaria ao maior grau de dependência ${ }^{24}$.

Identificou-se decréscimo da dependência nicotínica à medida que aumentou a escolaridade, em acordo com dados descritos anteriormente ${ }^{36}$. No Brasil, indivíduos com baixa escolaridade têm probabilidade cinco vezes maior de serem fuman- 
Tabela 4. Terapia medicamentosa utilizada nas sessões da Campanha Antitabágica em 11 idosos institucionalizados no "Parque Assistencial Jorge Cauhy", 2008.

\begin{tabular}{|c|c|c|c|c|c|}
\hline Pacientes & $\begin{array}{l}1^{\text {a }} \text { sessão } \\
\text { semanal }\end{array}$ & $\begin{array}{l}2^{\text {a }} \text { sessão } \\
\text { semanal }\end{array}$ & $\begin{array}{l}3^{\text {a }} \text { sessão } \\
\text { semanal }\end{array}$ & $\begin{array}{l}4^{\text {a }} \text { sessão } \\
\text { semanal }\end{array}$ & $\begin{array}{c}1^{\text {a }} \text { sessão de } \\
\text { manutenção quinzenal }\end{array}$ \\
\hline & \multicolumn{5}{|c|}{ Tratamento e ocorrências gerais } \\
\hline 5 & $\begin{array}{c}\text { TCC } \\
\text { Bupropiona }\end{array}$ & $\begin{array}{c}\text { TCC } \\
\text { Bupropiona }\end{array}$ & $\begin{array}{l}\text { Desistência } \\
\text { Intolerância à } \\
\text { abstinência. }\end{array}$ & & \\
\hline 6 & $\begin{array}{c}\text { TCC } \\
\text { Bupropiona }\end{array}$ & $\begin{array}{c}\text { TCC } \\
\text { Bupropiona } \\
\text { TRN }\end{array}$ & $\begin{array}{c}\text { TCC } \\
\text { Bupropiona } \\
\text { TRN }\end{array}$ & $\begin{array}{c}\text { TCC } \\
\text { Bupropiona } \\
\text { TRN suspensa: } \\
\text { voltou a fumar. }\end{array}$ & $\begin{array}{c}\text { TCC } \\
\text { Bupropiona }\end{array}$ \\
\hline 7 & $\begin{array}{c}\text { TCC } \\
\text { Bupropiona }\end{array}$ & $\begin{array}{c}\text { TCC } \\
\text { Bupropiona }\end{array}$ & $\begin{array}{l}\text { Desistência } \\
\text { Intolerância à } \\
\text { abstinência. }\end{array}$ & & \\
\hline 8 & $\begin{array}{c}\text { TCC } \\
\text { Bupropiona }\end{array}$ & $\begin{array}{c}\text { TCC } \\
\text { Bupropiona } \\
\text { TRN }\end{array}$ & $\begin{array}{c}\text { TCC } \\
\text { Bupropiona } \\
\text { TRN }\end{array}$ & $\begin{array}{c}\text { TCC } \\
\text { Bupropiona } \\
\text { TRN }\end{array}$ & $\begin{array}{c}\text { TCC } \\
\text { Bupropiona } \\
\text { TRN }\end{array}$ \\
\hline 9 & $\begin{array}{c}\text { TCC } \\
\text { Bupropiona }\end{array}$ & $\begin{array}{c}\text { TCC } \\
\text { Bupropiona }\end{array}$ & $\begin{array}{c}\text { TCC } \\
\text { Bupropiona } \\
\text { TRN }\end{array}$ & $\begin{array}{c}\text { TCC } \\
\text { Bupropiona } \\
\text { TRN suspensa: } \\
\text { voltou a fumar. }\end{array}$ & \\
\hline 12 & $\begin{array}{c}\text { TCC } \\
\text { Bupropiona }\end{array}$ & $\begin{array}{c}\text { TCC } \\
\text { Bupropiona } \\
\text { TRN }\end{array}$ & $\begin{array}{c}\text { TCC } \\
\text { Bupropiona } \\
\text { TRN }\end{array}$ & $\begin{array}{c}\text { TCC } \\
\text { Bupropiona } \\
\text { TRN }\end{array}$ & $\begin{array}{c}\text { TCC } \\
\text { Bupropiona } \\
\text { TRN }\end{array}$ \\
\hline 14 & $\begin{array}{c}\text { TCC } \\
\text { Bupropiona }\end{array}$ & $\begin{array}{c}\text { TCC } \\
\text { Bupropiona }\end{array}$ & $\begin{array}{c}\text { TCC } \\
\text { Bupropiona }\end{array}$ & $\begin{array}{l}\text { Desistência } \\
\text { Intolerância à } \\
\text { abstinência. }\end{array}$ & \\
\hline 18 & $\begin{array}{c}\text { TCC } \\
\text { Bupropiona } \\
\text { TCC }\end{array}$ & $\begin{array}{c}\text { TCC } \\
\text { Bupropiona } \\
\text { Desistência }\end{array}$ & $\begin{array}{l}\text { Desistência: } \\
\text { hospitalizado }\end{array}$ & & \\
\hline 19 & $\begin{array}{c}\text { TCC } \\
\text { Bupropiona }\end{array}$ & $\begin{array}{l}\text { Desistência } \\
\text { Intolerância à } \\
\text { abstinência. }\end{array}$ & & & \\
\hline 28 & $\begin{array}{c}\text { TCC } \\
\text { Bupropiona }\end{array}$ & $\begin{array}{c}\text { TCC } \\
\text { Bupropiona } \\
\text { TRN }\end{array}$ & $\begin{array}{c}\text { TCC } \\
\text { Bupropiona } \\
\text { TRN }\end{array}$ & $\begin{array}{c}\text { TCC } \\
\text { Bupropiona } \\
\text { Foi suspensa a } \\
\text { TRN suspensa: } \\
\text { voltou a fumar. }\end{array}$ & $\begin{array}{c}\text { TCC } \\
\text { Bupropiona }\end{array}$ \\
\hline 29 & $\begin{array}{c}\text { TCC } \\
\text { Bupropiona }\end{array}$ & $\begin{array}{c}\text { TCC } \\
\text { Bupropiona } \\
\text { TRN }\end{array}$ & $\begin{array}{c}\text { TCC } \\
\text { Bupropiona } \\
\text { TRN }\end{array}$ & $\begin{array}{c}\text { TCC } \\
\text { Bupropiona } \\
\text { TRN suspensa: } \\
\text { voltou a fumar. }\end{array}$ & $\begin{array}{c}\text { TCC } \\
\text { Bupropiona }\end{array}$ \\
\hline
\end{tabular}

TCC - Terapia Cognitivo Comportamental. TRN - Terapia de Reposição Nicotínica.

tes $^{35}$. Entretanto, Lima-Costa ${ }^{37}$ mostrou significativa associação do tabagismo à menor escolaridade somente nos indivíduos mais jovens, não surgindo este achado nos idosos. Para esse autor, a ausência de associação entre escolaridade e tabagismo nos indivíduos idosos é explicada como possível viés de sobrevivência, visto que se espera aumento da mortalidade precoce nos indivíduos fumantes com pior nível socioeconômico.
No estudo atual, encontrou-se provável depressão em $63 \%$ dos idosos tabagistas institucionalizados, predominando significativamente naqueles com maior grau de dependência nicotínica, corroborando o já demonstrado em outros estudos ${ }^{38,39}$. Esta comorbidade provavelmente dificultou o êxito da Campanha de Cessação Tabágica instituída, pois a depressão desenvolvida anteriormente ou durante a tentativa de ces- 
sação do fumo associa-se à menor chance de êxito durante o período de abstinência, assim como à maior frequência de recaídas ${ }^{40}$. Como a influência do tabagismo no aparecimento de depressão é controversa ${ }^{41}$, o achado do estudo atual serve de impulso para futuras pesquisas de cunho neurobiológico e epidemiológico, direcionadas à relação tabaco-depressão.

Naqueles indivíduos com elevada dependência nicotínica encontrou-se menor motivação para a cessação do tabagismo. Hughes ${ }^{42}$ relatou que os fumantes com elevada dependência podem apresentar baixa motivação, devido à falta de confiança em conseguir seu intento, achandose incapazes e com medo do sofrimento que a síndrome de abstinência produz, pois já tentaram e fracassaram outras vezes.

A Campanha de Cessação Tabágica efetuada na ILPI obteve somente $9 \%$ de êxito no período de um ano. Duncan et al..$^{43}$ relataram que, em idosos não institucionalizados, abordagens combinando aconselhamento comportamental e farmacoterapia alcançaram sucesso de $20 \%$ a $25 \%$ em um ano. Outros autores ${ }^{13,16}$ mostraram taxas de sucesso nos idosos não institucionalizados variando de $23 \%$ a $32 \%$ após um ano de cessação. Assim, quando se oferecem estratégias para que os idosos não institucionalizados abandonem o tabaco, a taxa de sucesso compara-se a dos jovens, apesar do ceticismo, fatalismo e diminuição de autoconfiança desta população. Burns ${ }^{16}$ mostrou até melhores resultados no grupo de idosos, afirmando que eles exibem maior probabilidade de sucesso, mesmo apresentando menor intenção de abandonar o cigarro se comparados aos jovens. Orleans et al. ${ }^{44}$ e Bratzler et al. ${ }^{15}$ sugeriram que os idosos não só fumam por período de tempo maior do que os tabagistas jovens, mas também estão menos cientes dos malefícios potenciais de uso do tabaco, apresentando como resultado, maior resistência a parar de fumar e menor número de tentativas de parar, tendendo a subestimar os riscos e a superestimar os benefícios de fumar.

O tabagismo aumenta o risco de patologia pulmonar, principalmente DPOC $^{45}$. No estudo atual, 79,3\% dos idosos tabagistas apresentaram distúrbio obstrutivo na espirometria, sendo $27,6 \%$ deles em grau severo. Como a maior parte dos fumantes inicia o vício na infância ou na adolescência, as doenças relacionadas ao tabagismo em geral surgem a partir dos 40 anos de idade, acentuando-se na velhice ${ }^{32}$.

A determinação da concentração de COex, que afere o hábito tabágico, é útil no monitora- mento de pacientes envolvidos em Programas de Cessação Tabágica. Santos et al..$^{28}$ encontraram, em fumantes, o COex médio de 14,01ppm e nos não fumantes de 2,50 ppm. Estes autores observaram que os valores de COex correlacionaramse positivamente com o número de cigarros fumados por dia e negativamente com o intervalo após ter fumado o último cigarro. No estudo atual, o COex médio nos idosos fumantes mostrou-se significativamente diferente nos três graus de gravidade do distúrbio obstrutivo, apresentando maior valor quanto mais grave o distúrbio obstrutivo, corroborando a correlação positiva entre o número de cigarros fumados por dia, os valores de COex encontrados e a gravidade da DPOC.

A abordagem antitabágica feita no idoso deve observar a maior possibilidade de comorbidades e risco aumentado de interações medicamen$\operatorname{tosas}^{31}$. É pertinente considerar que os indivíduos idosos frequentemente apresentam insuficiência dos sistemas excretores, levando à necessidade de correção nas doses dos medicamentos ${ }^{46}$. Deve-se estar atento à farmacodinâmica da TRN nos indivíduos com insuficiência renal, que apresentam sua eliminação prejudicada, o que pode levar ao aparecimento de efeitos colaterais. No estudo atual, não foi avaliada a função renal nos idosos, observando-se, entretanto, raros efeitos colaterais da TRN e somente em grau leve. Quanto à indicação da bupropiona nos idosos, é necessário refletir sobre seus riscos-benefícios em decorrência da alta prevalência de comorbidades e possíveis interações medicamentosas. No estudo atual, não surgiram efeitos colaterais com esta medicação.

Em indivíduos idosos, a abordagem terapêutica na cessação do vício tabágico deve-se adaptar às suas características, oferecendo-se apoio adequado de acordo com a fase de motivação apresentada, suas necessidades e seus perfis individuais ${ }^{46}$. Segundo Reichard et al. ${ }^{47}$, existem cinco perfis de adaptação dos idosos ao envelhecimento, sendo que aqueles com perfil maduro (idoso construtivo, independente) ou blindado (idoso rígido, defensivo) são os mais prováveis candidatos a aderirem, colaborarem e obterem êxito em Programas de Cessação Tabágica. Na maioria das vezes, mesmo considerando-se aptos, os idosos não se entregam aos novos desafios, pois têm dificuldade em romper barreiras e promover mudanças. Portanto, o reforço da autoeficácia é recurso da intervenção comportamental que deve ser usado à exaustão nesse grupo etário. Também, o aprendizado dos idosos é mais lento, o 
que requer reforço e detalhamento nas intervenções e no treinamento de habilidades ${ }^{48}$.

Para dar conta da complexidade do tabagismo, devem-se implementar medidas psicossociais, comparáveis ao tratamento farmacológico em termos de importância e de impacto ${ }^{49}$, pois a maioria das recaídas após período de abstinência de cigarros está relacionada a situações de estresse psicossocial ${ }^{50}$. Portanto, lidar com o dependente do tabaco requer a adoção de uma perspectiva genuinamente integrada, que inclua o biológico, o psicológico e o social, sem distinção de prioridade. No estudo atual, todas estas particularidades foram observadas cuidadosamente na Campanha instituída na ILPI.

Dois fatos contribuem para dificultar a abordagem ao idoso tabagista: aqueles aparentemente sadios utilizam o argumento de que já fumaram durante a vida inteira e não adoeceram e, portanto, estão imunes aos malefícios do cigarro; e, aqueles que já são portadores de doença argumentam que, pelo fato de já estarem doentes, não fará mais diferença se vierem a largar o tabaco $^{43}$. Frente a esses argumentos, deverão ser utilizados contra-argumentos que convençam o idoso sobre a qualidade de vida que o abandono do tabaco proporcionará à sua vida.

Pedroso e Oliveira ${ }^{51}$ acrescentaram outros argumentos que deverão ser listados para indivíduos idosos tabagistas: melhora de sua imagem entre os amigos, parentes e colegas; proteção dos filhos e da família contra os efeitos nocivos da fumaça do cigarro; não incomodar os outros; e ser segregado como fumante. Entretanto, para que se faça a abordagem com esses argumentos, é necessário conhecer os motivos pelos quais os idosos fumam e a influência dos ambientes familiar, socioeconômico e cultural sobre eles ${ }^{52}$. Portanto, estes argumentos não são válidos em população de idosos tabagistas institucionalizados.

A motivação individual é item dos mais relevantes na cessação definitiva do tabagismo, inter-relacionada com gama de variáveis hereditárias, psicológicas, fisiológicas e ambientais. $\mathrm{O}$ abandono do tabagismo resulta da determinação do indivíduo, conjuntamente com o apoio recebido (profissional, familiar, social e espiritual). Assim, a determinação do fumante de querer parar de fumar deve ser aliada ao apoio dos vários segmentos da sociedade, auxiliando-o de forma significativa no processo de abandono do tabagismo ${ }^{53}$.

Russo e Azevedo ${ }^{54}$ verificaram que a decisão de parar de fumar é principalmente influenciada por incentivo familiar. Vale ressaltar que a moti- vação do idoso fumante para deixar o tabagismo pode reforçar o despertar da consciência da sociedade contemporânea sobre a importância do seu papel saudável na família, como reservatório de sabedoria e de refúgio afetivo para crianças e jovens, assumindo a função de modelo de conduta para futuros adultos ${ }^{45}$. Contudo, questiona-se a importância desse papel entre os idosos tabagistas institucionalizados, visto que sua interação com a família, bem como com a sociedade, está limitada à convivência interna nas ILPI. Assim, acredita-se que várias dessas particularidades interferiram negativamente na Campanha de Cessação Tabágica efetuada na ILPI, levando ao baixo índice de sucesso encontrado.

O tema tabagismo entre os idosos internados em ILPI é complexo, ajudando-os muitas vezes a encontrar forças para enfrentar e superar as muitas dificuldades do viver, como sua fragilidade e sua solidão. Nesta população, o cigarro torna-se importante apoio no enfrentamento dos embates da vida, cobrando, porém, alto preço pelo amparo e prazer proporcionados. Questiona-se se fatores como ausência de vínculo afetivo, depressão, menor requisição social e médica para deixar de fumar, oportunidades reduzidas, falta de atividade produtiva e insegurança quanto à autoeficácia, contribuíram como reforço positivo ao tabagismo nos idosos institucionalizados estudados. Estes idosos tinham menos laços afetivos, sendo descrito que aqueles que mantém algum nível de vínculo afetivo, não necessariamente o marital, são menos dependentes da nicotina ${ }^{34}$. Parece que a institucionalização pode contribuir na perpetuação do tabagismo, visto as relações de abandono familiar, os maus tratos, a negligência dos profissionais de saúde e a consequente solidão que tal população, frequentemente, enfrenta nesses locais.

Considera-se este estudo como um despertar para a realidade do tabagismo em idosos internados em ILPI, pois o consumo de tabaco constitui problema de saúde pública nesta população ${ }^{34}$, tendo-se obtido baixa taxa de sucesso com a Campanha de Cessação Tabágica instituída entre eles. Deve-se incentivar a participação de outras faixas etárias nesses grupos, favorecendo maior interação intergeracional e, consequentemente, maior dinamismo e enriquecimento do repertório temático, o que poderá propiciar maior êxito desses Programas.

Para obtenção de sucesso nos Programas de Cessação Tabágica feitos em ILPI, há necessidade de ampliação da rede de relacionamentos, vínculos afetivos e relações de interdependência entre 
os idosos tabagistas institucionalizados. Concluise, assim, que as atuais estratégias assistenciais para o enfrentamento do tabagismo divulgadas por meio de consensos e políticas governamentais, por vezes, desconsideram, reduzem ou simplificam as complexas dimensões da vida humana. Escapam a esse modelo, de forma fugaz, tanto a subjetividade dos sujeitos implicados quanto as determinações socioeconômicas e culturais que perpassam as singularidades humanas. Deve-se questionar e repensar nossos olhares e posturas, se ao invés de simplesmente retirar o cigarro pretende-se efetivamente apoiar os idosos tabagistas institucionalizados, no esforço para se libertarem desta dependência que acarreta tão graves implicações à sua vida e à sua saúde.

\section{Colaboradores}

AA Carvalho, L Gomes, AL Loureiro e AJC Bezerra participaram igualmente de todas as etapas de elaboração do artigo.

\section{Referências}

1. Reichert J, Araújo AJ, Gonçalves CMC, Godoy I, Chatkin JM, Sales MPU, Santos SRRA. Diretrizes para cessação do tabagismo - 2008. J Bras Pneumol 2008; 34(10):845-880.

2. Halty LS, Hünttner MD, Oliveira Netto I, Fenker T, Pasqualini T, Lempek B, Santos A, Muniz A. Pesquisa sobre tabagismo entre médicos de Rio Grande, RS: prevalência e perfil do fumante. J Bras Pneumol 2002; 28(2):77-83.

3. Lima-Costa MF, Barreto SM, Uchoa E, Firmo JOA, Vidigal PG, Guerra HL. The Bambuí Health and Aging Study (BHAS): prevalence of risk factors and use of preventive health care services. Rev Panam Salud Publica 2001; 9(4):219-226.

4. Marinho V, Laks J, Coutinho ESF, Blay SL. Tobacco use among the elderly: a systematic review and metaanalysis. Cad Saude Publica 2010; 26(12):2213-2233.

5. Peixoto SV, Firmo JOA, Lima CMF. Health conditions and smoking among older adults in two communities in Brazil (The Bambuí and Belo Horizonte Health Surveys). Cad Saude Publica 2006; 22(9):1925-1934.

6. Instituto Nacional de Câncer (INCA). Inquérito domiciliar sobre comportamento de risco e morbidade referida de doenças e agravos não transmissíveis Brasil, 15 capitais e Distrito Federal 2002-2003. Rio de Janeiro: INCA; 2004

7. Husten CG, Shelton DM, Chrismon JH, Lin YW, Mowery P, Powell FA. Cigarette smoking and smoking cessation among older adults: United States, 1965-94. Tob Control 1997; 6(3):175-180.

8. Gerontology. Older Persons - Tobacco's Forgotten Victims. Tobacco \& The Elderly Projects. Michigan. 1998. [acessado 2010 maio 18]. Disponível em: http:/ /www.tcsg.org/tobacco/spring98/contentssp98. htm

9. Halty LS. Abordagem de grupos especiais: idosos. Bras Pneumol 2004; 30(2):58-61. 
10. Ostbye T, Taylor DH, Jung SH. A longitudinal study of the effects of tobacco smoking and other modifiable risk factors of ill health in middle-aged and old Americans: results from the Health and Retirement Study and Asset and Health Dynamics among the Oldest Old Survey. Prev Med 2002; 34(3):334-345.

11. Menotti A, Mulder I, Nissinen A, Feskens E, Giampaoli S, Tervahauta M, Kromhout D. Cardiovascular risk factors and 10-year all-cause mortality in elderly European male population: the FINE study. Eur Heart J 2001; 22(7):573-579.

12. Sachs DPL. Cigarette smoking: health effects and cessation strategies. Clin Geriatr Med 1986; 2(2):337362.

13. Appel DW, Aldrich TK. Smoking cessation in the elderly. Clin Geriatr Med 2003; 19(1):77-100.

14. Jarvis MJ, Wardle J, Waller J, Owen I. Prevalence of hardcore smoking in England, and associated attitudes and beliefs: cross sectional study. BMJ 2003; 326(7398):1061-1065.

15. Bratzler DW, Oehlert WH, Austelle A. Smoking in the elderly: it's never too late to quit. J Okla State Med Assoc 2002; 95(3):185-191.

16. Burns DM. Cigarette smoking among the elderly: disease consequences and the benefits of cessation. Am J Health Promot 2000; 14(6):357-361.

17. Lantz SM. Smoking cessation in the older. Clin Geriatr 2002; 10(9):26-32.

18. Davim RMB, Torres GV, Dantas SMM, Lima VM. Estudo com idosos de instituições asilares no município de Natal/RN: características socioeconômicas e de saúde. Rev Lat. Am. Enfermagem 2004; 12(3):518-524.

19. Pavarini SCI. Dependência comportamental na velhice: uma análise do cuidado prestado ao idoso institucionalizado [tese]. Campinas: Faculdade de Educação, Unicamp; 1996.
20. Folstein MF, Folstein SE, Mchugh PR. "Mini-mental state". A practical method for grading the cognitive state of patients for the clinician. J Psychiatr Res 1975; 12(3):189-198.

21. Brucki SMD, Nitrini R, Carameli P, Bertolucci PHF, Okamoto IH. Sugestões para o uso do mini-exame do estado mental no Brasil. Arq Neuropsiquiatr 2003; 61(3B):777-781.

22. Yesavage JA. Differential diagnosis between depression and dementia. Am J Med 1993; 94(Supl. 5A):23S$28 \mathrm{~S}$.

23. Almeida OP, Almeida SA. Confiabilidade da versão brasileira da Escala de Depressão em Geriatria (GDS) versão reduzida. Arq Neuro-Psiquiatr 1999; 57(2B):421-426.

24. Fagerström KO. Measuring degree of physical dependence to tobacco smoking with reference to individualization of treatment. Addict Behav 1978; 3(34):235-241.

25. Meneses-Gaya IC, Zuardi AW, Loureiro SR, Crippa JA. Psychometric properties of the Fagerström test for nicotine dependence. J Bras Pneumol 2009; 35(1):73-82.

26. Pereira CAC, Barreto SP, Simões JG, Pereira FWL, Gerstler JG, Nakatani J. Valores de referência para espirometria em uma amostra da população brasileira adulta. J Bras Pneumol 1992; 18(1):10-22.

27. Sociedade Brasileira de Pneumologia e Tisiologia SBPT. Diretrizes para Cessação do Tabagismo. J Bras Pneumol 2004; 30(Supl. 2):S1-S76.

28. Santos UP, Gannam S, Abe JM, Esteves PB, Freitas Filho M, Wakassa TB, Issa JS, Terra Filho M, Stelmach R, Cukier A. Emprego da determinação de monóxido de carbono no ar exalado para a detecção do consumo de tabaco. J Pneumol 2001; 27(5): 231-236. 
29. Viegas CAA, Reichert J. Diretrizes para cessação do tabagismo. J Bras Pneumol 2004; 30(Suppl. 2): S36-S40.

30. Benowitzs NL. Pharmacology of nicotine: addiction and therapeutics. Annu Rev Pharmacol Toxicol 1996; 36(April):597-613.

31. Fiore MC, Bailey WC, Cohen SJ, Dorfman SF, Golstein MG, Gritz ER, Heyman RB, Holbrook J, Jaen CR, Kottke TE, Lando HA, Mecklenburg R, Mullen PD, Nett LM, Robinson L, Stitzer ML, Tommasello AC, Villejo L, Wewers ME. Smoking cessation. Smoking cessation guideline panel. In: US Department of Health and Human Services, Public Health Service, Agency for Health Care Policy and Research: Clinical Practice Guideline 2000; 18(9606292).

32. Instituto Nacional de Câncer (INCA). Coordenação de Prevenção e Vigilância. Prevalência de tabagismo no Brasil: Dados dos inquéritos epidemiológicos em capitais brasileiras. Rio de Janeiro: INCA; 2004.

33. Gonçalves LG, Vieira ST, Siqueira FV, Hallal PC. Prevalência de quedas em idosos asilados do município de Rio Grande. Rev Saude Publica 2005; 42(5):938-945.

34. Carvalho AA, Vianna LG, Viegas CA, Loureiro AL. Tabagismo em idosos internados em Instituições de Longa Permanência de Brasília, Distrito Federal. I Bras Pneumol 2010; 36(3):339-346.

35. Organização Pan-Americana da Saúde (OPAS). Tabaco e pobreza, um círculo vicioso - a convençãoquadro de controle do tabaco: uma resposta. Brasília: Ministério da Saúde (MS); 2004.

36. Silva GA, Valente JG, Almeida LM, Moura EC, Malta. DC. Tabagismo e escolaridade no Brasil 2006. Rev Saude Publica 2009; 43(Supl. 2):S48-S56.

37. Lima-Costa MF. A escolaridade afeta, igualmente, comportamentos prejudiciais à saúde de idosos e adultos mais jovens? Inquérito de Saúde da Região Metropolitana de Belo Horizonte, Minas Gerais, Brasil. Epidemiol Serv Saúde 2004; 13(4):201-208.

38. Lando H, Hennrikus D, McCarty M, Vessey J. Predictors of quitting in hospitalized smokers. Nicotine Tob Res 2003; 5(2):215-222.

39. Lagrue G, Dupont P, Fakhfakh R. Anxiety and depressive disorders in tobacco dependence. Encephale $2002 ; 28(4): 374-377$

40. Glassman AH, Covey LS, Stetner F, Rivelli S. Smoking cessation and the course of major depression: a follow-up study. Lancet 2001; 357(9272):1929-1932.

41. Leventhal AM, Ramsey SE, Brown RA, LaChance HR, Kahler CW. Dimensions of depressive symptoms and smoking cessation. Nicotine Tob Res 2008; 10(3):507-517.
42. Hughes JR. Why does smoking so often produce dependence? A somewhat different view. Tob Control 2001; 10(Supl. 1):62-64.

43. Duncan BB, Schmidt MI, Giugliani ERJ. Medicina ambulatorial: Condutas de atenção primária baseadas em evidências. 3a Edição. Porto Alegre: Artmed; 2006.

44. Orleans CT, Jepson C, Resch N, Rimer BK. Quitting motives and barriers among older smokers: the 1986 adult use of tobacco survey revisited. Cancer 1994, 74(Supl. 7):2055-2061.

45. Cataldo JK. Smoking and aging. Clinical implications. Part I: Health and consequence. J Gerontol Nurs 2003; 29(9):15-20.

46. Instituto Nacional de Câncer (INCA). Consenso: Abordagem e Tratamento do Fumante - 2001. Rio de Janeiro: INCA; 2001.

47. Reichard S, Livson F, Peterson PG. Aging and Personality. New York: Wiley; 1962.

48. Mohiuddin SM, Mooss AN, Hunter CB, Grollmes TL, Cloutier DA, Hilleman DE. Intensive smoking cessation intervention reduces mortality in highrisk smokers with cardiovascular disease. Chest 2007 131(2):446-452.

49. Sutherland G. Smoking: can we really make a difference? Heart 2003; 89(Supl. II):1125-1127.

50. Presman S, Carneiro E, Gigliotti A. Tratamentos não farmacológicos para o tabagismo. Rev Psiquiatr Clin 2005; 32(5):267-275

51. Pedroso ERP, Oliveira RG. Blackbook - Clinica Médica. 1a Edição. Belo Horizonte: Blackbook; 2007.

52. Silva Filho OA. Tabagismo em idosos. [acessado 2010 set 15]. Disponível em: http://www.tabagismoonline. com.br/entendendomelhor/gerontologia/tabag.em. idosos

53. Echer IC, Barreto SSM. Determination and support as successful factors for smoking cessation. Rev Latino-Am Enfermagem 2008; 16(3):445-451.

54. Russo AC, Azevedo RCS. Fatores motivacionais que contribuem para a busca de tratamento ambulatorial para a cessação do tabagismo em um hospital geral universitário. J Bras Pneumol 2010; 36(5):603-611.

Artigo apresentado em 31/10/2011

Aprovado em 27/01/2012

Versão final apresentada em 01/02/2012. 